\title{
Verteilung und Valenz der Kationen in Spinellsystemen mit Eisen und Vanadium, I Röntgenographische und Mößbauer-spektroskopische Untersuchung des Spinellsystems $\mathrm{ZnV}_{2} \mathrm{O}_{4}-\mathrm{ZnFe}_{2} \mathbf{0}_{4}$
}

\author{
Distribution and Valence of the Cations in Spinel Systems with Iron and Vanadium, I \\ $\mathrm{X}$-ray Investigation and Mössbauer Spectra of the Spinel System $\mathrm{ZnV}_{2} \mathrm{O}_{4}-\mathrm{ZnFe}_{2} \mathrm{O}_{4}$ \\ Erwin Riedel*, Till Dützmann und Norbert Pfeil \\ Institut für Anorganische und Analytische Chemie der Technischen Universität Berlin, \\ Straße des 17. Juni 135, D-1000 Berlin 12 \\ Z. Naturforsch. 35b, 1257-1260 (1980); eingegangen am 2. Juni 1980 \\ Zinc-Iron-Vanadium Spinels, Mössbauer Spectroscopy, Valence Distribution \\ The spinel system $\mathrm{Zn}\left[\mathrm{Fe}_{x} \mathrm{~V}_{2-x}\right] \mathrm{O}_{4}$ has been prepared by heating mixtures of $\mathrm{ZnV}_{2} \mathrm{O}_{4}$ \\ and $\mathrm{ZnFe}_{2} \mathrm{O}_{4}$ at $1000^{\circ} \mathrm{C}$. The lattice constants increase linearly with $x$ by $3.6 \mathrm{pm}$, the \\ oxygen parameters are constant with a mean value $u=0.384_{8}$. The parameters of room \\ temperature Mössbauer spectra show that in the whole series $\mathrm{V}$ (III) and $\mathrm{Fe}(\mathrm{III})$ are \\ present.
}

\section{Einleitung}

Die Mischkristallreihe aus dem normalen Spinell $\mathrm{Fe}^{2+}\left[\mathrm{V}_{2}{ }^{3+}\right] \mathrm{O}_{4}$ und dem inversen Spinell

$\mathrm{Fe}^{3+}\left[\mathrm{Fe}^{2+} \mathrm{Fe}^{3+}\right] \mathrm{O}_{4}$ wurde bereits mehrfach untersucht [1-12]. Die Ionenladungen und die Kationenverteilungen in dieser Mischkristallreihe $\mathrm{Fe}\left[\mathrm{Fe}_{x} \mathrm{~V}_{2-x}\right] \mathrm{O}_{4}$ sind durch die Lage der beiden elektronischen Gleichgewichte

$$
\begin{aligned}
& \mathrm{Fe}_{\mathrm{O}^{3+}}+\mathrm{V}_{\mathrm{O}^{3+}} \rightleftharpoons \mathrm{Fe}_{\mathrm{O}^{2+}}+\mathrm{V}_{\mathrm{O}^{4+}} \\
& \mathrm{Fe}_{\mathrm{T}}{ }^{2+}+\mathrm{Fe}_{\mathrm{O}^{3+}} \rightleftharpoons \mathrm{Fe}_{\mathrm{T}}{ }^{3+}+\mathrm{Fe}_{\mathrm{O}^{2+}}
\end{aligned}
$$

bestimmt. Während über $\mathrm{Fe}_{3} \mathrm{O}_{4}$ in der Literatur einheitliche Auffassungen bestehen, wurden für die Mischkristalle unterschiedliche Verteilungen diskutiert. Beispielsweise wurden zur Erklärung der Eigenschaften von $\mathrm{Fe}_{2} \mathrm{VO}_{4}$ sämtliche nach den Gleichgewichten mögliche Verteilungen herangezogen:

$$
\begin{aligned}
& \mathrm{Fe}^{2+}\left[\mathrm{Fe}^{2+} \mathrm{V}^{4+}\right] \mathrm{O}_{4}[4,5] \text { oder } \\
& \mathrm{Fe}_{1-\lambda}{ }^{2+} \mathrm{Fe}_{\lambda}{ }^{3+}\left[\mathrm{Fe}_{\lambda}^{2+}{ }^{2+} \mathrm{Fe}_{1-\lambda}{ }^{3+} \mathrm{V}^{3+}\right] \mathrm{O}_{4}
\end{aligned}
$$

mit $\lambda=0$ (normal) [8], $\lambda=1$ (invers) [9-11] oder $0<\lambda<1$ (teilweise invers) $[6,7,12]$.

Zur Klärung der Gleichgewichtslage in Spinellsystemen, die Eisen und Vanadium enthalten, wurden übersichtliche Modellsysteme hergestellt und untersucht, über die das folgende Schema einen Überblick gibt.

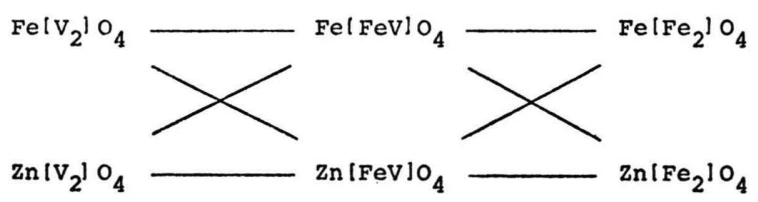

Im I. Teil der Mitteilungsreihe wird über Untersuchungen der Valenzverteilung in der Mischkristallreihe $\mathrm{Zn}\left[\mathrm{V}_{2}\right] \mathrm{O}_{4}-\mathrm{Zn}\left[\mathrm{Fe}_{2}\right] \mathrm{O}_{4}$ mit der MößbauerSpektroskopie berichtet. Die Gitterkonstanten, Seebeck-Koeffizienten und IR-Spektren des Systems sind bereits gemessen worden [25]. Das MößbauerSpektrum von $\mathrm{ZnFe}_{2} \mathrm{O}_{4}$ wurde schon mehrfach gemessen und die Quadrupolaufspaltung berechnet $[13,14]$. In früheren Untersuchungen $[15,16]$ konnte durch Messung des Seebeck-Koeffizienten am Spinell $\mathrm{ZnFeVO}_{4}$, in den $\mathrm{Fe}^{2+}$-Ionen bzw. $\mathrm{V}^{4+}$-Ionen eingebaut wurden, die Valenzverteilung

$\mathrm{Zn}\left[\mathrm{Fe}^{3+} \mathrm{V}^{3+}\right] \mathrm{O}_{4}$ ermittelt werden. Für die Energie des Elektronenübergangs $\mathrm{Fe}^{3+}+\mathrm{V}^{3+} \rightarrow \mathrm{Fe}^{2+}+\mathrm{V}^{4+}$ wurde $0,35 \mathrm{eV}$ bestimmt.

\section{Untersuchungsmethoden}

Die Untersuchungsmethoden werden an dieser Stelle für die gesamte Mitteilungsreihe zusammenfassend beschrieben.

Die Röntgenuntersuchung erfolgte mit einer Guinier-deWolff-Kamera ( $\mathrm{CuK}_{\alpha}$-Strahlung) und mit einem Philips-Diffraktometer ( $\mathrm{CrK}_{\alpha}$-Strahlung). Als Eichsubstanz wurde Goldpulver benutzt. Als röntgenographisch phasenrein betrachtet werden Substanzen, bei denen die stärksten Fremdreflexe der Goniometeraufnahmen weniger als $0,5 \%$ der Intensität des stärksten Spinellreflexes besitzen. Die Gitterkonstante wurde aus Diffraktometermessun-

\footnotetext{
* Sonderdruckanforderungen an Prof. Dr. E. Riedel. 0340-5087/80/1000-1257/\$01.00/0
} 
gen unter Verwendung des Nelson-Riley-Extrapolationsverfahrens oder aus Guinieraufnahmen bestimmt. Die Anionenparameter und die Kationenverteilung wurden aus dem Minimum des Gütefaktors $\mathrm{R}=\left[\Sigma|| \mathrm{F}_{\text {exp }}|-| \mathrm{F}_{\mathrm{ber}}|| \Sigma\left|\mathrm{F}_{\text {ber }}\right|\right] 100[\%]$ ermittelt

IR-Spektren wurden bei Raumtemperatur von $200 \mathrm{~cm}^{-1}$ bis $1000 \mathrm{~cm}^{-1}$ mit einem IR 325 von Perkin-Elmer aufgenommen.

Die ${ }^{57} \mathrm{Fe}-M o ̈ ß b a u e r-S p e k t r e n$ wurden mit einem Spektrometer AME-30 Comp. (Elscint) in Transmission aufgenommen. Die Geschwindigkeit (symmetrische Dreiecksform) der Quelle, ${ }^{57} \mathrm{Co}$ in $\mathrm{Rh}$ oder in Pd, wurde durch simultane Messung der Absolutgeschwindigkeit mit einem Laser-Interferometer LI-1 (Elscint) oder eines Eisenspektrums geeicht. Probenspektrum und Eichspektrum wurden jeweils in 500 Kanälen gespeichert. Als Detektor wurde ein $\mathrm{NaI}(\mathrm{Tl})$-Szintillationszähler (Harshaw) benutzt; der Fe-Gehalt des Be-Eingangsfensters wurde beim Fitten des Spektrums berücksichtigt. Für Hochtemperaturspektren stand ein evakuierbarer Ofen MF-2A (Elscint) und ein Regelgerät TC-4B (Ricor) zur Verfügung. Der Druck in der Heizkammer betrug $10^{-8}$ bar bis $10^{-9}$ bar, die Temperaturkonstanz $\pm 0,02 \mathrm{~K}$. Bei Raumtemperatur wurden zylindrische Plexiglasprobenträger (Innendurchmesser $25 \mathrm{~mm}$ ), für Hochtemperaturmessungen Bornitridprobenträger benutzt. Der Eisengehalt der Absorber betrug maximal $10 \mathrm{mg} / \mathrm{cm}^{2}$. Die Auswertung des Spektrums erfolgte mit einem leastsquares-fit-Programm unter Annahme von LorentzFunktionen und gleicher Intensität und Halbwertsbreite der Dublettkomponenten. Als Gütekriterium des Fits wird die Größe $\chi^{2}=\sum_{1}^{500}\left(\mathrm{Y}_{\exp }-\mathrm{Y}_{\text {ber }}\right)^{2} / \mathrm{Y}_{\exp }$ berechnet. Der statistische Fehler der Isomerieverschiebung gut aufgelöster Linien beträgt weniger als $\pm 0,001 \mathrm{~mm} / \mathrm{s}$. Alle Isomerieverschiebungen werden relativ zu Eisen angegeben [17]. Bei den abgebildeten Spektren sind die Meßwerte als Striche mit der Länge der doppelten Standardabweichung, die berechneten Absorptionslinien gepunktet und ihre Summation durchgezogen dargestellt.

Die integrale Thermospannung $\mathrm{E}$ wurde an unkontaktierten Sinterkörpern zwischen $20^{\circ} \mathrm{C}$ und $80^{\circ} \mathrm{C}$ mit einem Keithley-Voltmeter (616 Digital Elektrometer) gemessen. Der Seebeck-Koeffizient wurde aus der Steigung der Funktion $E=f(t)$ bestimmt.
Das Reduktionsvermögen und der Vanadiumgehalt wurden durch Titration mit $\mathrm{KMnO}_{4}$ - bzw. $\mathrm{FeSO}_{4}$-Lösung kontrolliert [18].

\section{Präparation}

Die Substanzen der Reihe $\mathrm{ZnFe}_{x} \mathrm{~V}_{2_{-}} \mathrm{O}_{4}$ wurden aus Mischungen der Spinelle $\mathrm{ZnV}_{2} \mathrm{O}_{4}$ und $\mathrm{ZnFe}_{2} \mathrm{O}_{4}$ bei $1000{ }^{\circ} \mathrm{C}$ unter Ölpumpenvakuum hergestellt. $\mathrm{ZnV}_{2} \mathrm{O}_{4}$ wurde aus $\mathrm{ZnO}$ und $\mathrm{V}_{2} \mathrm{O}_{3}$ bei $900{ }^{\circ} \mathrm{C}$ unter Ölpumpenvakuum, $\mathrm{ZnFe}_{2} \mathrm{O}_{4}$ aus $\mathrm{ZnO}$ und $\mathrm{Fe}_{2} \mathrm{O}_{3}$ bei $1000{ }^{\circ} \mathrm{C}$ an Luft präpariert. Die Reaktionszeiten betrugen zwei bis dreimal ein bis drei Tage. $\mathrm{ZnV}_{2} \mathrm{O}_{4}$ wurde zusätzlich bei $900{ }^{\circ} \mathrm{C}$ unter einer $\mathrm{CO}_{2}-\mathrm{CO}$. Atmosphäre bei Sauerstoffdrücken zwischen $10^{-15}$ bar und $10^{-11}$ bar gesintert. Bei Sauerstoffpartialdrücken von $10^{-12}$ bar bis $10^{-13}$ bar entstehen Produkte, deren Guinieraufnahmen nur noch Spinellreflexe zeigen und deren Seebeck-Koeffizienten Maximalwerte von etwa $600 \mu \mathrm{V} / \mathrm{K}$ erreichen.

\section{Röntgenographische Untersuchung}

Die Gitterkonstante der Spinellmischkristallreihe $\mathrm{Zn}\left[\mathrm{Fe}_{x} \mathrm{~V}_{2_{-} x}\right] \mathrm{O}_{4}$ nimmt bei Substitution von Vanadium durch Eisen linear um 3,6 pm zu (Tab. I).

Tab. I. Gitterkonstanten im Spinellsystem $\mathrm{ZnFe}_{x} \mathrm{~V}_{2-x} \mathrm{O}_{4}$.

\begin{tabular}{llllll}
\hline$x$ & $a[\mathrm{pm}]$ & $x$ & $a[\mathrm{pm}]$ & $x$ & $a[\mathrm{pm}]$ \\
\hline 0,0 & 840,8 & 0,2 & 841,5 & 1,8 & 843,9 \\
& 841,3 & 0,3 & 842,0 & 2,0 & 844,4 \\
0,025 & 840,8 & & 841,4 & & 844,5 \\
& 841,0 & 0,5 & 841,7 & {$[24]$} & 841,0 \\
0,05 & 841,0 & 0,75 & 842,3 & {$[13]$} & 841,6 \\
0,075 & 841,1 & 0,8 & 842,0 & {$[14]$} & 843,0 \\
0,1 & 841,0 & 1,0 & 842,5 & {$[25]$} & 843,6 \\
& 841,1 & 1,2 & 842,9 & {$[26]$} & 844,11 \\
0,15 & 841,2 & 1,4 & 843,0 & {$[27]$} & 844,6 \\
\hline
\end{tabular}

Bemerkenswert ist, daß die in der Literatur angegebenen Gitterkonstanten von $\mathrm{ZnFe}_{2} \mathrm{O}_{4}$ wesentlich stärker differieren als der methodische Fehler erwarten läßt. Der Sauerstoffparameter ist annähernd konstant; der Mittelwert beträgt $\mathrm{u}=0,3848 \pm$ 0,0005. Mit den Beziehungen

$$
\begin{aligned}
(\mathrm{Me}-\mathrm{O})_{\mathrm{T}} & =a \sqrt{3}(1 / 8+\delta) \\
(\mathrm{Me}-\mathrm{O})_{\mathrm{o}} & =a\left(1 / 16-\delta / 2+3 \delta^{2}\right)^{0,5} \\
\mathrm{u} & =0,375+\delta
\end{aligned}
$$

und dem Radius $\mathrm{r}^{\mathrm{IV}} \mathrm{O}^{2-}=138 \mathrm{pm}$ [19] wurden die in Tab. II angegebenen Ionenradien berechnet. Die 
Differenzen zu den Radien von Shannon und Prewitt [19], sowie zu den aus den Spinellsystemen $\mathrm{Zn}^{2+}\left[\mathrm{Me}_{2}{ }^{3+}\right] \mathrm{O}_{4}[20]$ und $\mathrm{Me}^{2+}\left[\mathrm{V}_{2}{ }^{3+}\right] \mathrm{O}_{4}$ [21] berechneten Radien, sind kleiner als 1 pm.
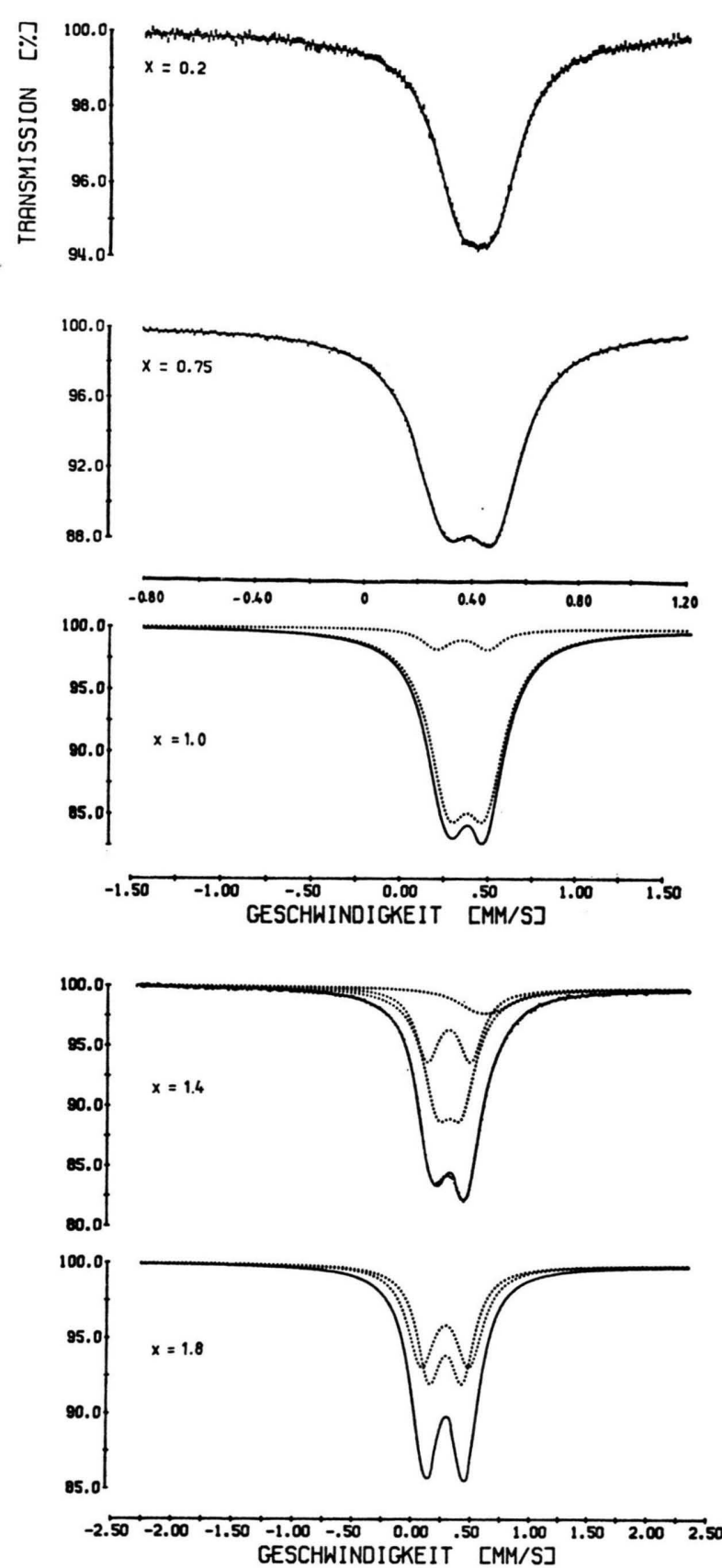

Abb. 1. Mößbauer-Spektren des Systems $\mathrm{Zn}\left[\mathrm{F}_{x} \mathrm{~V}_{2-x}\right] \mathrm{O}_{4}$.
Tab. II. Ionenradien von $\mathrm{Zn}^{2+}, \mathrm{V}^{3+}$ und $\mathrm{Fe}^{3+}$ (in pm).

\begin{tabular}{llll}
\hline & $\begin{array}{l}\text { Radien aus } \\
\text { dem System } \\
\mathrm{Zn}\left[\mathrm{Fe}_{x} \mathrm{~V}_{2-x}\right] \mathrm{O}_{4}\end{array}$ & $\begin{array}{l}\text { Radien } \\
\text { nach } \\
\text { Shannon } \\
{[19]}\end{array}$ & $\begin{array}{l}\text { Radien aus den } \\
\text { Systemen } \\
\mathrm{Zn}^{2+}\left[\mathrm{Me}_{2}{ }^{3+}\right] \mathrm{O}_{4}[20] \\
\text { und } \\
\mathrm{Me}^{2+}\left[\mathrm{V}_{2}{ }^{3+}\right] \mathrm{O}_{4}[21]\end{array}$ \\
\hline & & & 58,7 \\
${\text { Iv } \mathrm{Zn}^{2+}}^{\text {VIV }^{3+}}$ & $58,7 \pm 0,3$ & 60 & $54,3 \pm 0,3$ \\
VIFe $^{3+}$ & $65,2 \pm 0,5$ & 64,0 & 64,4 \\
\hline
\end{tabular}

\section{Mößbauer-Spektren}

Obwohl im System $\mathrm{Zn}\left[\mathrm{Fe}_{x} \mathrm{~V}_{2-x}\right] \mathrm{O}_{4}$ alle Eisenionen oktaedrisch koordiniert sind, bestehen die Raumtemperatur-Mößbauer-Spektren (Abb. 1, Tab. III) nur im Bereich $x \lesssim 0,3$ aus einem einzigen Dublett. Bei größeren $x$-Werten sind wegen der Asymmetrie der Gesamtabsorption zwei Dubletts mit nahezu identischer Isomerieverschiebung, aber unterschiedlicher Quadrupolaufspaltung, zur Beschreibung erforderlich. Die Asymmetrie kann durch die Anisotropie des Debye-Waller-Faktors verursacht sein [22]. Die mittlere Isomerieverschiebung nimmt linear von $\bar{\delta}=0,39_{9} \mathrm{~mm} / \mathrm{s} \quad(x=0,025)$ auf $\bar{\delta}=$ $0,34_{3} \mathrm{~mm} / \mathrm{s}(x=2) \mathrm{ab}$, die mittlere Quadrupolaufspaltung nimmt von $\bar{\Delta}=0,11 \mathrm{~mm} / \mathrm{s} \quad(x=0,025)$

Tab. III. Mößbauer-Parameter im Spinellsystem $\mathrm{Zn}\left[\mathrm{Fe}_{x} \mathrm{~V}_{2-x}\right] \mathrm{O}_{4}$.

\begin{tabular}{|c|c|c|c|c|c|}
\hline$x$ & FA & $\begin{array}{l}\delta[\mathrm{mm} / \mathrm{s}] \\
\delta[\mathrm{mm} / \mathrm{s}]\end{array}$ & $\begin{array}{l}\frac{\Delta}{\Delta}[\mathrm{mm} / \mathrm{s}] \\
{[\mathrm{mm} / \mathrm{s}]}\end{array}$ & $\Gamma[\mathrm{mm} / \mathrm{s}]$ & $\chi^{2}$ \\
\hline 0,025 & 1 & $0,39_{9}$ & 0,11 & 0,20 & 546 \\
\hline 0,2 & 1 & $0,39_{8}$ & 0,13 & 0,23 & 476 \\
\hline 0,3 & 1 & $0,21_{6}$ & 0,14 & 0,24 & 493 \\
\hline 0,75 & 1 & $0,20_{8}$ & 0,19 & 0,26 & 838 \\
\hline \multirow{3}{*}{1,0} & 0,91 & $0,38_{0}$ & 0,21 & 0,27 & \multirow[t]{3}{*}{463} \\
\hline & \multirow[t]{2}{*}{0,09} & $0,34_{5}$ & 0,29 & \multirow[t]{2}{*}{0,19} & \\
\hline & & $0,37_{7}$ & 0,21 & & \\
\hline \multirow[t]{4}{*}{1,2} & 0,43 & 0,389 & 0,21 & 0,25 & \multirow[t]{4}{*}{485} \\
\hline & 0,52 & $0,35_{7}$ & 0,26 & 0,26 & \\
\hline & \multirow[t]{2}{*}{0,05} & 0,75 & 0,17 & \multirow[t]{2}{*}{0,45} & \\
\hline & & $0,39_{0}$ & 0,23 & & \\
\hline \multirow[t]{4}{*}{1,4} & 0,29 & $0,38_{2}$ & 0,15 & 0,26 & \multirow[t]{4}{*}{548} \\
\hline & 0,60 & $0,37_{2}$ & 0,32 & 0,27 & \\
\hline & \multirow[t]{2}{*}{0,11} & 0,68 & 0,15 & \multirow[t]{2}{*}{0,43} & \\
\hline & & $0,40_{8}$ & 0,25 & & \\
\hline \multirow[t]{4}{*}{1,6} & 0,51 & $0,37_{0}$ & 0,23 & 0,29 & \multirow[t]{4}{*}{524} \\
\hline & 0,43 & $0,35_{7}$ & 0,29 & 0,24 & \\
\hline & \multirow[t]{2}{*}{0,06} & 0,73 & 0,19 & \multirow[t]{2}{*}{0,42} & \\
\hline & & $0,39_{0}$ & 0,25 & & \\
\hline \multirow{3}{*}{1,8} & 0,51 & $0,35_{1}$ & 0,40 & 0,28 & \multirow[t]{3}{*}{456} \\
\hline & \multirow[t]{2}{*}{0,49} & $0,35_{6}$ & 0,28 & \multirow[t]{2}{*}{0,25} & \\
\hline & & $0,35_{3}$ & 0,34 & & \\
\hline \multirow[t]{3}{*}{2,0} & 0,55 & $0,34_{4}$ & 0,47 & \multirow{3}{*}{$\begin{array}{l}0,28 \\
0,23\end{array}$} & \multirow[t]{3}{*}{573} \\
\hline & \multirow[t]{2}{*}{0,45} & $0,34_{2}$ & 0,32 & & \\
\hline & & $0,34_{3}$ & 0,40 & & \\
\hline
\end{tabular}




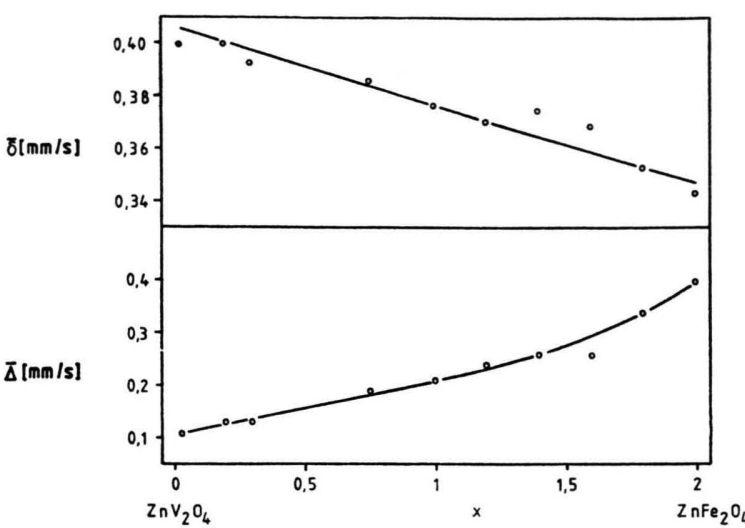

Abb. 2. Mittlere Isomerieverschiebungen und mittlere Quadrupolaufspaltungen im System $\mathrm{Zn}\left[\mathrm{Fe}_{x} \mathrm{~V}_{2-x}\right] \mathrm{O}_{4}$.

auf $\bar{\Delta}=0,40 \mathrm{~mm} / \mathrm{s}(x=2)$ stetig zu (Abb. 2). Die Mößbauer-Parameter zeigen, daß in der gesamten Mischkristallreihe $\mathrm{Fe}^{3+}$ - neben $\mathrm{V}^{3+}$-Ionen vorliegen. Für oktaedrisch koordinierte $\mathrm{Fe}^{2+}$-Ionen wäre ein größerer Shift und eine größere Aufspaltung zu erwarten. Beispielsweise findet man beim $\mathrm{Ge}\left[\mathrm{Fe}_{2}\right] \mathrm{O}_{4}$ $\delta=1,10 \mathrm{~mm} / \mathrm{s}$ und $\Delta=2,85 \mathrm{~mm} / \mathrm{s}[23]$.

Die Spektren im Bereich $1,2 \leqq x \leqq 1,6$ zeigen zusätzlich schwache Absorptionen im Flankenbereich, die durch ein weiteres Dublett mit anomal großer Halbwertsbreite berücksichtigt wurden. Den Isomerieverschiebungen von $0,68 \mathrm{~mm} / \mathrm{s}$ bis $0,75 \mathrm{~mm} / \mathrm{s}$ entsprechen Eisenionen der mittleren Oxidationsstufe $+2,4$ bis 2,5, wenn man eine lineare Änderung des Shifts mit der Oxidationsstufe voraussetzt. Als Ursache muß angenommen werden, daß Elektronenaustausch zwischen $2 \%-3 \% \mathrm{Fe}^{2+}$-Ionen und $\mathrm{Fe}^{3+}$-Ionen stattfindet. Die Entstehung von $\mathrm{Fe}(\mathrm{II})$ ist jedoch unklar.

Übereinstimmend ergeben Mößbauer-Spektren und die früher gemessenen Seebeck-Koeffizienten $[15,16]$, daß im Spinellsystem $\mathrm{Zn}\left(\mathrm{Fe}_{x} \mathrm{~V}_{2-x}\right] \mathrm{O}_{4}$ das Redoxgleichgewicht $\mathrm{Fe}_{\mathrm{O}}{ }^{3+}+\mathrm{V}_{\mathrm{O}}{ }^{3+} \rightleftharpoons \mathrm{Fe}_{\mathrm{o}}{ }^{2+}+\mathrm{V}_{\mathrm{O}^{4+}}$ auf der Seite von $\mathrm{Fe}$ (III) und V(III) liegt.
[1] M. P. Gupta und H. B. Mathur, J. Phys. C 8, 370 (1975).

[2] P. de V. du Plessis, J. Phys. C: Solid St. Phys. 4, 2919 (1971).

[3] M. Lensen, Ann. Chimie 4, 891 (1959).

[4] D. B. Rogers, R. J. Arnott, A. Wold und J. B. Goodenough, J. Phys. Chem. Solids 24, 347 (1963).

[5] M. J. Rossiter, J. Phys. Chem. Solids 26, 775 (1965).

[6] E. Riedel, Dissertation, TU Berlin 1965.

[7] B. N. Varskoi, A. N. Il'ina und N. V. Gogareva, Russ. J. Phys. Chem. 40, 447 (1966).

[8] S. K. Banerjee, W. O'Reilly, T. C. Gibb und N. N. Greenwood, J. Phys. Chem. Solids 28, 1323 (1967).

[9] J.-C. Bernier und P. Poix, Ann. Chim. 2, 81 (1967).

[10] M. Wakihara, Y. Shumizu und T. Katsura, J. Solid State Chem. 3, 478 (1971).

[11] M. Abe, M. Kawachi und S. Nomura, J. Solid State Chem. 10, 351 (1974).

[12] J. D. Lee und D. Schroer, J. Phys. Chem. Solids 37, 739 (1976).

[13] A. Hudson und H. J. Whitfield, Md. Phys. 12, 165 (1967).
[14] B. J. Evans, S. S. Hafner und H. P. Weber, J. Chem. Phys. 55, 5282 (1971).

[15] E. Riedel, G. Colsmann und B. Reuter, Ber. Bunsenges. Phys. Chem. 72, 880 (1968).

[16] G. Colsmann, B. Reuter und E. Riedel, Z. Anorg. Allg. Chem. 401, 41 (1973).

[17] J. G. Stevens, V. E. Stevens und A. Hilger, Mössbauer Effect Data Index, London 1974.

[18] B. Reuter, J. Jaskowsky und W. Laqua, Z. Anal. Chem. 214, 185 (1965).

[19] R. D. Shannon und C. T. Prewitt, Acta Crystallogr. B 25, 306 (1969).

[20] E. Riedel, Habilitationsschrift, TU Berlin 1970.

[21] B. Reuter und E. Riedel, Z. Anorg. Allg. Chem. 369, 306 (1969).

[22] N. N. Greenwood und T. C. Gibb, Mössbauer Spectroscopy, Chapman and Hall Ltd., London 1971.

[23] N. Pfeil, Dissertation, TU Berlin 1978.

[24] C. M. Yagnik und H. B. Mathur, Md. Phys. 16, 625 (1969).

[25] G. Colsmann, Dissertation, TU Berlin 1970.

[26] Ed. L. G. Berry; Powder Diffraction File, Joint Committee on Powder Diffraction Standards, Swarthmore, U.S.A. 1976.

[27] W. Paterno, Diplomarbeit, TU Berlin 1974. 